\title{
Aplicación de la metodología Scrum en la evaluación de competencias transversales
}

\author{
Jordi Capó Vicedo $^{\mathrm{a}}$, Amparo Bonet ${ }^{\mathrm{b}}$, Antonio Giménez-Morera ${ }^{\mathrm{c}}$
}

${ }^{a}$ Centre for Research in Business Management (CEGEA), Universitat Politècnica de València, Spain. jorcavi0@esp.upv.es, ${ }^{\mathrm{b}}$ Universitat Politècnica de València, Department of Economy and Social Sciences, Camino de Vera s/n, 46022, Valencia, Spain, ${ }^{c}$ Universitat Politècnica de València, Department of Economy and Social Sciences, Camino de Vera s/n, 46022, Valencia, Spain angimo1@doctor.upv.es.

\begin{abstract}
With the incorporation of transversal and specific competences to check the learning degree of Higher Education students, a new scenario is opened in which it is necessary to raise and apply new methodologies to be able to verify the success in the acquisition of these competences.

This presentation presents the results obtained in the application of an active methodology, such as the scrum, in the assessment of transversal competences in a subject of the Master's Degree in Business Administration and Management taught at the Escuela Politécnica de Alcoi of the Universitat Politècnica de València.
\end{abstract}

Keywords: transversal competences, assessment, scrum, higher education.

\footnotetext{
Resumen

Con la incorporación de las competencias, tanto transversales como especificas, para comprobar el grado de aprendizaje por parte de los alumnos de la Enseñanza Superior, se abre un nuevo escenario en el que es necesario plantear y aplicar nuevas metodologías para poder comprobar el éxito en la adquisición de dichas competencias.

En esta ponencia se presentan los resultados obtenidos en la aplicación de una metodología activa, como es el scrum en la evaluación de las competencias transversales de una asignatura del Máster Universitario en Dirección y Administración de Empresas, impartida en la Escuela Politécnica de Alcoi de la Universitat Politècnica de València.
}

Palabras clave: competencias transversales, evaluación, scrum, enseñanza superior. 


\section{Introducción}

Con el proceso de convergencia hacia el Espacio Europeo de Enseñanza Superior (EEES), la universidad española se ha planteado la necesidad de abordar una serie de cambios que permitan una transición de un modelo de enseñanza en el que el aprendizaje de los alumnos no se planteaba como el objetivo principal a un modelo en el que se busca precisamente el aprendizaje permanente y efectivo por parte del alumnado.

En este contexto, era necesario emprender la incorporación de nuevas metodologías en las que los alumnos pasasen a ser los actores principales y que les permitieran adquirir y afianzar todas las competencias, tanto específicas como transversales, necesarias para el posterior desarrollo de sus profesiones.

De esta forma, la aplicación de estas metodologías permite que los alumnos pasen de ser meros oyentes a participar plenamente en el desarrollo de las clases permitan un aprendizaje más eficiente por parte de los alumnos.

En este sentido, con la incorporación de las competencias, tanto transversales como específicas para comprobar el grado de aprendizaje por parte de los alumnos de la Enseñanza Superior, se abre un nuevo escenario en el que es necesario plantear y aplicar nuevas metodologías para poder comprobar el éxito en la adquisición de dichas competencias.

En la presente ponencia, se presentan los resultados obtenidos de la aplicación de la metodología Scrum en la evaluación de dos competencias transversales en una asignatura del Máster Universitario en Dirección y Administración de Empresas impartida en la Escuela Politécnica de Alcoi de la Universitat Politècnica de València.

\section{Antecedentes}

Con el nuevo escenario que se presenta como consecuencia de la incorporación al EEES, los alumnos deben ser capaces de enfrentarse y abordar con éxito situaciones reales y no únicamente superar un examen teórico.

Por tanto, es necesario que los alumnos sean capaces de adquirir habilidades y competencias que les permitan desarrollar los resultados de su aprendizaje. No obstante, para poder lograr estos objetivos es necesario aplicar nuevas metodologías en las que el alumnado se involucre de una forma más activa y que permitan un conocimiento más aplicado en forma de adquisición de competencias, tal y como De la Cruz (2003) afirma, el aprendizaje de competencias consiste en conocer, comprender y utilizar pertinentemente.

Estas competencias, las podemos agrupar entre aquellas que son específicas de un título académico o una profesión y aquellas que son más genéricas o transversales, las cuales son habilidades necesarias e importantes para el desarrollo de cualquier profesión, pero no se pueden encontrar de forma explícita en el desarrollo de asignaturas específicas.

En concreto, en la Universitat Politècnica de València, se han definido 13 competencias transversales a partir de las cuales se intentan cubrir los diferentes aspectos que recogen 
diferentes organismos como la agencia ABET, EUR-ACE, además de los reales decretos españoles:

- CT-01. Comprensión e integración

- CT-02. Aplicación y pensamiento práctico

- CT-03. Análisis y resolución de problemas

- CT-04. Innovación, creatividad y emprendimiento

- CT-05. Diseño y proyecto

- CT-06. Trabajo en equipo y liderazgo

- CT-07. Responsabilidad ética, medioambiental y profesional

- CT-08. Comunicación efectiva

- CT-09. Pensamiento crítico

- CT-10. Conocimiento de problemas contemporáneos

- CT-11. Aprendizaje permanente

- CT-12. Planificación y gestión del tiempo

- CT-13. Instrumental específica

De estas 13 competencias, en el presente trabajo se analizarán los resultados obtenidos en la evaluación de 2 de ellas en la asignatura de Responsabilidad Social Corporativa del Máster Oficial en Dirección de Empresas. En concreto se trata de:

- CT-06. Trabajo en equipo y liderazgo.

- CT-12. Planificación y gestión del tiempo.

\subsection{La asignatura Responsabilidad Social Corporativa}

La asignatura de Responsabilidad Social Corporativa se enmarca dentro del segundo curso del máster oficial en dirección de empresas (MUDE) que se imparte en el campus de Alcoi de la Universitat Politècnica de València. Es una asignatura optativa de 4,5 créditos del itinerario de dirección corporativa correspondiente al primer cuatrimestre.

Durante el curso 2017/18 habían 26 alumnos matriculados en el MUDE, de los cuáles 8 estaban matriculados en la asignatura de Responsabilidad Social Corporativa.

Los objetivos de la asignatura son: por una parte, conocer los conceptos básicos y las herramientas de Responsabilidad Social Corporativa (RSC) presentes en el mundo empresarial y, por otra, aportar los conocimientos teóricos y prácticos para la gestión de una empresa que quiera ser socialmente responsable. 
La integración de la Responsabilidad Social Corporativa en sus estrategias empresariales puede suponer una serie de ventajas y de beneficios a largo plazo. Es en este punto dónde se hace más hincapié en la asignatura, con el fin de que los alumnos sean capaces de ver la importancia de este enfoque integrado de la gestión, lo que supone un modelo de gobierno basado en la sostenibilidad y la gestión de las externalidades empresariales en lo económico, social y medioambiental contempladas a un mismo nivel.

La asignatura se estructura en 6 unidades didácticas con un enfoque eminentemente práctico, con clases participativas y la realización y análisis de casos prácticos complementarios a la teoría, así como de trabajos de búsqueda y análisis de información específica.

Tabla 1. Datos descriptivos de la asignatura

\begin{tabular}{cccc}
\hline Asignatura & Titulación & Departamento & Alumnos \\
\hline $\begin{array}{c}\text { Responsabilidad Social } \\
\text { Corporativa }\end{array}$ & $\begin{array}{c}\text { Máster universitario en } \\
\text { Dirección de Empresas }\end{array}$ & $\begin{array}{c}\text { Economía y } \\
\text { Ciencias Sociales }\end{array}$ & 8 \\
\hline Centro & Créditos & Curso & Duración \\
\hline $\begin{array}{c}\text { Escuela Politécnica } \\
\text { Superior de Alcoi }\end{array}$ & 4,5 & $2^{\circ}$ & Cuatrimestral \\
\hline
\end{tabular}

Fuente: Elaboración propia

\section{Metodología}

Para la medición de la adquisición de las competencias transversales comentadas en los puntos anteriores, se decidió emplear una metodología basada en el trabajo en grupo. De esta forma, se pueden lograr resultados cognitivos más altos que con el trabajo individual (Johnson, Johnson y Satnne, 2000) y, además, permite que los diferentes componentes del equipo aporten sus propias destrezas y puntos de vista de forma que se complementes entre sí (Crooks, 1988).

En concreto, se empleó la metodología Scrum, que se detalla a continuación.

\subsection{Scrum}

La metodología Scrum surgió en la industria de desarrollo de software con el fin de crear un marco de trabajo que permitiera el desarrollo ágil de proyectos. Sin embargo, la flexibilidad y adaptabilidad de esta metodología, así como la sencillez para ser aplicada, ha contribuído a que su aplicación se haya extendido a diversos contextos entre los cuales, cada vez más, está el educativo.

Se trata de una metodología de desarrollo ágil en la que se llevan a cabo una serie de ciclos breves para el desarrollo de los proyectos denominados "Sprints". 
Cada sprint se desarrolla en tres fases: una reunión de planificación en la que se marca el objetivo del Sprint y se organizan las diferentes tareas del equipo de forma que este trabaje de forma conjunta, un período de trabajo en las que se realizan las reuniones de seguimiento, y una reunión de revisión al final del sprint en el que se revisa el incremento en el proyecto. Por último, se lleva a cabo una reunión de evaluación del trabajo de cara a poder mejorar.

Cada uno de los ciclos o sprints tiene un tiempo limitado y se espera que, al final de cada uno de ellos, se haya generado una mayor funcionalidad del proyecto.

Además, dentro del marco de trabajo es necesario establecer diferentes equipos formados por varios alumnos. Cada uno de los equipos Scrum se compone de un responsable del producto (dueño del producto, Product Owner), el equipo de desarrollo (Development Team o Scrum Team) y el Scrum Master, que es el responsable de asegurar que todo se entiende y se aplica de forma correcta.

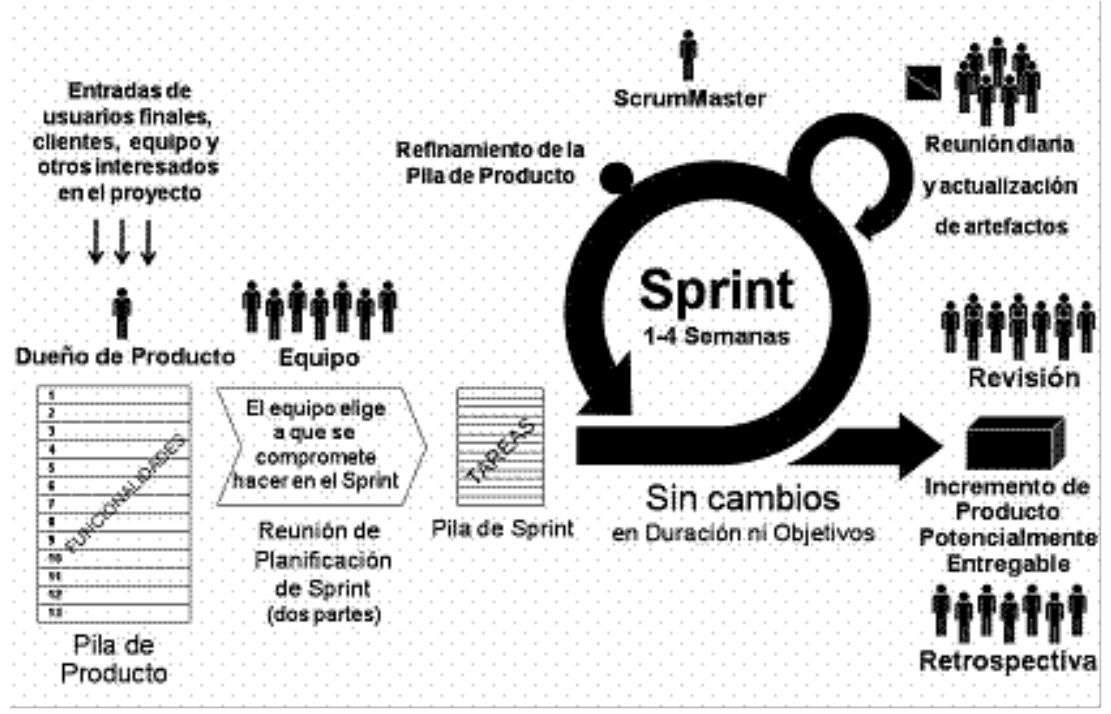

Figura 1. Roles, artefactos y eventos principales de SCRUM (Fuente: Deemer et al., 2009)

\subsection{Aplicación de la metodología}

En el caso de la asignatura de Responsabilidad Social Corporativa, al ser 8 alumnos se hicieron dos equipos de 4 personas entre las cuales se repartieron los diferentes roles y tareas.

Una vez organizados los equipos y los roles, se explicó el proyecto que debían realizar los alumnos. En concreto, cada equipo debía seleccionar una empresa y analizarla desde el punto de vista de la integración e incorporación de los principios de la RSC en sus políticas internas y en la gestión diaria de las mismas. Una vez analizadas, a partir de las conclusiones obtenidas, los alumnos debían ser capaces de plantear un conjunto de medidas 
encaminadas a mejorar la gestión socialmente responsable de las empresas, entre las cuales se integraría la realización de una memoria de sostenibilidad.

Para la realización del proyecto se plantearon llevar a cabo 4 Sprints con una duración de tres semanas cada uno.

$\mathrm{Al}$ término de cada uno de los sprints, cada grupo debía preparar y presentar un documento a partir de las reuniones de revisión y de retrospectiva de Sprint que se habían llevado a cabo., De ese modo, se debe poder valorar la evolución y la implicación de cada grupo en el proyecto.

Para la valoración de las diferentes capacidades involucradas en la asignatura, se valoró tanto la presentación de una memoria final y su exposición, como la participación de las diferentes fases (reuniones) del proyecto y la involucración de los miembros de cada equipo.

En concreto, de acuerdo con los objetivos marcados en el proyecto institucional de la Universitat Politècnica de Valènica, para lograr alcanzar el nivel de dominio de la competencia transversal 6 (Trabajo en equipo y liderazgo), se debe: "Participar y colaborar activamente en las tareas del equipo, orientándose hacia el trabajo en común”.

La consecución de esos objetivos se puede valorar a través de los siguientes indicadores: Indicadores:

- Aceptar y cumplir los objetivos del equipo.

- Acudir y participar activamente en las reuniones del equipo.

- Realizar las tareas asignadas dentro del equipo en el plazo fijado.

En este caso, la aplicación de la metodología Scrum, favoreció la motivación y el compromiso de los diferentes integrantes de cada equipo Scrum, de forma que los diferentes equipos trabajaron de forma conjunta, ayudando a desarrollar sus capacidades y alcanzando el nivel deseado de la competencia en cuestión.

En cuanto a la competencia transversal 12 "Planificación y gestión del tiempo", para lograr el nivel de dominio se debe ser capaz de "Planificar las actividades a realizar a corto y medio plazo".

Los indicadores para valorar el grado de cumplimiento son:

- Definir los objetivos a cumplir a corto o medio plazo.

- Determinar las actividades a desarrollar a medio plazo, jerarquizándolas en función de su importancia.

- Asignar tiempos a las actividades y realizarlas con el formato requerido.

- Analizar el desarrollo de las actividades.

En este caso, la aplicación de la metodología permitió la organización de las tareas y planificación de las mismas, puesto que la propia naturaleza del scrum ya conlleva la 
planificación y organización del trabajo en los sprints en las que se organizan las tareas y se revisa el desarrollo y consecución de objetivos, por lo que permitió alcanzar el nivel adecuado en la competencia en cuestión.

\section{Resultados}

Con respecto a los resultados obtenidos en la asignatura durante el curso 2017/18, se detallan a continuación.

En cuanto al rendimiento académico, la nota media obtenida por los alumnos utilizando la metodología Scrum fue de 8,7 sobre 10, mientras que en los cursos anteriores en los que no se utilizó esta metodología, la nota media fue de 6,7 sobre 10 , produciéndose un incremento significativo del rendimiento.

Con respecto a las competencias transversales, tal y como se comenta a continuación, también se ha apreciado una clara mejoría de acuerdo con la clasificación de adquisición de competencias que sigue la siguiente escala: A - Excelente; B - Adecuado; C - en desarrollo; D - no alcanzado.

En la competencia transversal 6 "Trabajo en equipo y liderazgo", la aplicación de la metodología permitió que los diferentes integrantes de los equipos trabajaran de forma conjunta, poniendo en común los avances así como las dificultades que iban encontrando. De esta forma, el nivel de consecución de la competencia durante este curso fue de un $60 \%$ A y un $40 \%$ B, frente a un $42 \%$ A y $58 \%$ B de otros cursos.

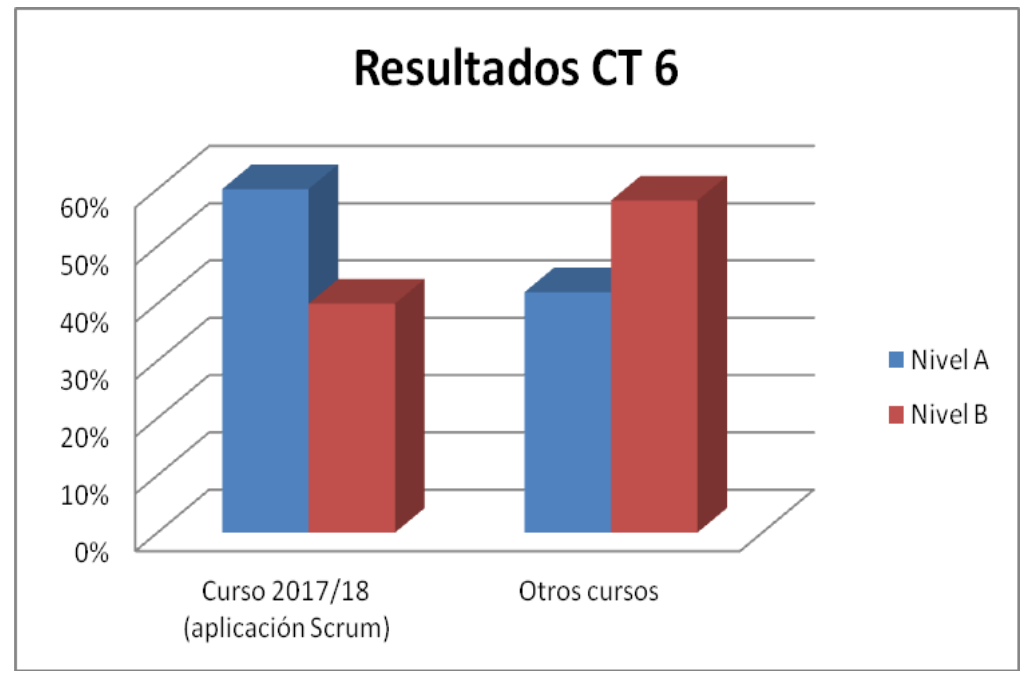

Figura 2. Resultados nivel alcanzado en la competencia transversal 6.

En cuanto a la competencia transversal 12 "Planificación y gestión del tiempo", la organización del proyecto en los sprints, con las entregas periódicas y la planificación, tanto de las tareas individuales como grupales, permitió pasar de un nivel de un 31\% A y $69 \%$ B de otros años a un $61 \%$ A y $39 \%$ B. 


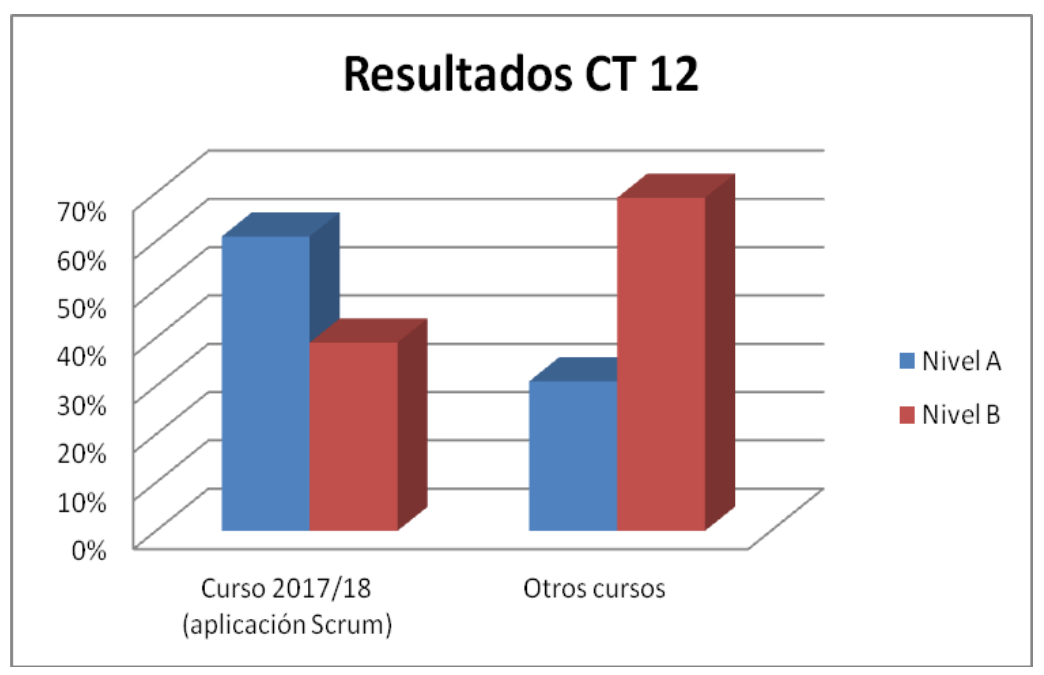

Figura 3. Resultados nivel alcanzado en la competencia transversal 12.

\section{Conclusiones}

Con la incorporación de las competencias tanto transversales como específicas para comprobar el grado de aprendizaje por parte de los alumnos universitarios, también se incorpora la necesidad de aplicar nuevas metodologías más activas que permitan un aprendizaje continuo y eficiente.

En el caso objeto de la presente ponencia, en el que se planteaba la aplicación de una metodología de trabajo colectivo como es el scrum, los resultados obtenidos han sido muy positivos puesto que han permitido que los alumnos se involucraran y participaran más activamente en la asignatura, pudiendo desarrollar sus habilidades y consiguiendo, al final del cuatrimestre, alcanzar los niveles de dominio de las competencias transversales analizadas.

No obstante, hay que tener en cuenta que, en este caso, el número de alumnos de la asignatura era bastante reducido, con sólo 8 alumnos. Por tanto, la realización de las reuniones como el seguimiento y tutorización de las mismas pudo realizarse de forma mucho más minuciosa, lo cual puede ser considerado como una limitación en el caso de aplicarse en grupos mucho más grandes.

\section{Referencias}

Crooks, T. J. (1988). The impact of classroom evaluation practices on students, Review of Educational Research, 58, 438-481.

Deemer, P. et al. (2009). Información básica de Scrum (The Scrum Primer). San Francisco. Recuperado de http://www.goodagile.com/Scrumprimer/Scrumprimer es.pdf 
De La Cruz Tomé, M. A. (2003). "Necesidad y objetivos de la formación pedagógica del profesor Universitario", Revista de Educación, 331, 55-66.

Johnson, D.W., Johnson, R.T. y Stanne, M.B. (2000). Cooperative Learning methods: A MetaAnálysis. Cooperative Learning Center at the University of Minnesota. Recuperado de http://www.clcrc.com/pages/cl-methods.hml

Schwaber ,K. y Sutherland, J. (2013). The Scrum Guide. Recuperado de https://www.Scrum.org/Scrum-Guide

Universitat Politècnica de València (2019). Competencias transversales. Recuperado de http://www.upv.es/contenidos/COMPTRAN/ 